\title{
To control or not to control, that is the question
}

\author{
Charlene E. Gamaldo, MD, FAASM ${ }^{\mathrm{a}}$, Alyssa Gamaldo, $\mathrm{PhD}^{\mathrm{b}}$, Justin C. McArthur, MBBS, \\ MPH, FAAN ${ }^{a, c}$, and Michael T. Smith, PhD ${ }^{d}$
}

aDepartment of Neurology, Johns Hopkins University, Baltimore, Maryland bSchool of Aging Studies, University of South Florida, Tampa, Florida 'Departments of Pathology, Epidemiology, and Medicine, Johns Hopkins University, Baltimore, Maryland dDepartment of Psychiatry and Behavioral Medicine, Johns Hopkins University, Baltimore, Maryland

\begin{abstract}
The reviewer points out correctly that we used a correlational approach and therefore cannot determine causality or establish the direction of the significant relationships observed between some of the sleep indices and cognitive performance in the HIV-positive individuals. Given that this is one of the first studies exploring how sleep in HIV-positive individuals might impact cognitive performance, we believe that it is an acceptable scientific contribution to conduct an exploratory analysis to highlight any potential relationships. If there are acceptable relationships, then, as the reviewer mentions, one could expand and explore the underlying mechanisms for this relationship using an experimental approach. This paper illustrates possible relationships between cognition and sleep in HIV participants. As mentioned in the manuscript, we hope that future studies will be conducted that can confirm and/or expand upon these findings.
\end{abstract}

This paper represents the third paper generated from a protocol that included HIV seropositive and seronegative participants. As discussed in the methods section, more specific detail regarding the full protocol and methods has been published previously. This includes the protocol for measuring sleep based on well-established methods and validated tools including in lab polysomnography, 2 week ambulatory actigraphy monitoring, electronic sleep diaries and clinical sleep evaluations conducted by sleep medicine trained experts. ${ }^{1,2,3}$ The reviewer's suggestions regarding the merit of including a control group in this subgroup analysis paper raises an issue that the scientific community is now beginning to openly discuss. This issue has been raised in other disciplines, such as cognitive aging, particularly when exploring cross-ethnic groups in cognitive performance. For example, in the cognitive aging literature, researchers have often explored cognitive function in minority samples by conducting cross-group research, in which a control group of white participants is included in the study. However, some researchers have proposed that these studies do not appreciate the unique cognitive attributes of the minority group of interest. ${ }^{4,5}$ Furthermore, they do not account for factors that may explain the within-group variability in cognitive function often observed in minority groups. ${ }^{6}$ As a result, these researchers suggest that within-group research is a more reliable and valid approach than cross-group research for identifying the underlying sources of poor cognitive functioning within minority samples, and subsequently, explaining the racial group differences in cognitive performance often reported in previous literature. This same theoretical perspective could also be applied to clinical samples, such as our HIV sample. Cognitive deficits have been long recognized as a being prevalent in HIV seropositive individuals, even those who are aviremic. Indeed, the

Conflicts of Interest: There are no conflicts of interest. 
NeuroAIDS research community is actively searching for underlying pathophysiologic mechanisms, despite virological stability on cART. Furthermore, the cognitive functioning and/or deficits are likely to vary greatly within a diverse HIV cohort much less between seropositive HIV subjects and seronegative "controls." The within-group approach will be useful in identifying the factors that explain the within-group variability in cognitive function observed in HIV samples, and, subsequently, will assist researchers in understanding the mechanisms for cognitive deficits within HIV individuals. The crossgroup approach of making comparisons between a HIV sample and non-HIV control sample would ignore this within-group variability and would not adequately address the nature of variability in cognitive performance among the HIV individuals. As a result, a cross-group approach may limit the potential for identifying mechanisms for the cognitive deficits observed in HIV individuals and strategies of intervention for this population. In summary we appreciate the interest in our paper. As highlighted by the reviewer's comments, we believe that the overarching message is the need for further investigations into this relationship; as it certainly encompasses a complex inter-relationship both physiologically, environmentally, and socio-culturally.

\section{Acknowledgments}

We thank Dr. Kawada for his thoughtful review to our submission and provide this response.

Financial Support: Funded by NIH grant \#5P30MH075673-S02; UL1 RR 025005.

\section{References}

1. Gamaldo CE, Spira AP, Hock RS, et al. Sleep, function and HIV: A multi-method assessment. AIDS Behav. 2013

2. Gamaldo CE, Gamaldo A, Creighton J, et al. Sleep and cognition in an HIV+ cohort: A multimethod approach. J Acquir Immune Defic Syndr. 2013

3. Gamaldo CE, McArthur JC. The evaluation and diagnosis of 'insomnia' in relation to sleep disturbance prevalence and impact in early-treated HIV-infected persons. Clin Infect Dis. 2012

4. Aiken-Morgan, AT.; Whitfield, KE.; Paige, MA. Correlates of cognitive aging in racial/ethnic minorities. In: Whitfield, KE.; Baker, TA., editors. Handbook of Minority Aging. New York, NY: Springer Publishing Compay; 2013. p. 85-101.

5. Markides, KS.; Liang, J.; Jackson, JS. Race, ethnicity, and aging: Conceptual and methodological issues. In: Binstock, RH.; George, LK., editors. Handbook of aging and social sciences. New York, NY: Van Nostrand Reinhold; 1990. p. 112-129.

6. Whitfield KE, Baker-Thomas T. Individual differences in aging minorities. International Journal of Aging \& Human Development. 1999; 48:73-79. [PubMed: 10363561] Whitfield KE, Allaire JC, Belue R, Edwards CL. Are comparisons the answer to understanding behavioral aspects of aging in racial and ethnic groups? Journal of Geronotology: Psychological Sciences. 2008; 63B:301-308. 